\title{
IR Based Auto-Recharging System for Autonomous Mobile Robot
}

\author{
M. V. Sreenivas Rao ${ }^{1 *}$, M.Shivakumar \\ ${ }^{1}$ Dept. of Electronics and Instrumentation Engineering, Visveswaraya Technological University, Belgavi, Karnataka, India. \\ ${ }^{2}$ GSSSIETW, Mysuru. Karnataka. India \\ Email: ${ }^{1}$ mvsraogsss@gmail.com \\ *Corresponding Author
}

\begin{abstract}
As autonomous mobile robots are progressively utilized for appropriated missions, a significant issue that should be tackled is the autonomous recharging problem. The robots can be recharged by planning and arranging effectively to maximize its working efficiency. This paper presents the implementation of automatic docking robot with docking strategy and recharging capabilities. The robot is programmed using an algorithm which will guide the robot to move around in a square path of 30 inch by 30 inch continuously. While the robot is performing its assigned task, the battery remaining voltage is monitored by voltage detection module. When the battery voltage reaches threshold value of less than $12 \mathrm{~V}$, the microcontroller commands the robot to go back to the docking station for recharging autonomously. This system uses IR receiver sensor in front of the robot and IR transmitter sensor near docking station. The active IR transmitter sensor which transmit infrared signal located near docking area serves as landmark in guiding robot towards docking area. The robot scans the transmitted IR signal from the sensor transmitter only when it needs to charge its battery, if detected it will take the path of charging station. Once the robot approaches the charging station with the required orientation, it connects to the supply terminals for charging. The data related to battery charging voltage is transmitted by microcontroller through Bluetooth HC-05 to PLX DAQ software tool in PC stores it in the Excel sheet as the data arrive. Once the battery is fully charged the robot moves back to continue its original task.
\end{abstract}

Keywords- Autonomous mobile robot, IR sensor, Docking station and $P L X D A Q$ software.

\section{INTRODUCTION}

In recent times, autonomous mobile robots are extensively employed in the fields such as security systems, cleaning large buildings, office automation, dangerous environment detection, warehouse and distribution, hospital services, life sciences applications and so on. When the mobile robot is executing its task continuously, energy gets sourced uninterruptedly from the batteries.

The key issue is to detect the instant at which voltage level of the battery reaches threshold value and directing the robot to execute auto recharging process. The mobile robot searches for the docking station using range sensors which serves as landmark in guiding robot towards docking area. Once the docking station is detected, the mobile robot gets docked autonomously for recharging.

The system employed in the current work consists of four wheeled autonomous mobile robots and a docking station. A docking technique based on scan by the robot for the IR sensor transmitter to move towards the docking station is proposed. The docking station consists of T-shape wooden structure having flat interface surface mounted with metallic electrical contacts. Once the robot starts charging at the docking station, Bluetooth present in the mobile robot communicates with the computer to record the data related to charging voltage using PLX-DAQ software tool. The robot having the capabilities of automatic docking and battery recharging is presented in this paper.

This paper is organized as follows: Section II discusses about survey of scholarly sources about current knowledge including substantive findings in the area of current work. Section III introduces the methodology and overall architecture of the autonomous robot and the docking station employed in the current work. The locomotion mechanism and the docking method are presented. The experimental results on docking performance of the robot and battery charging data are given in Section IV. Concluding remarks are given in Section V.

\section{RELATED WORK}

K. Varun Raj et al have developed docking system which assists the robot in such a way that the docking target forms the midpoint of the line joining the beacons. Active infrared beacons are used by the proposed docking system to transmit signals around the workspace. The robot using onboard sensors detects target beacons successfully in getting docked [1].

Guangming Song et al have proposed surveillance robot for home security. The robot communicates with docking station using zigbee. Based on self-localization technique and infrared detectors, the robot locates and reaches docking station for recharging [2]. Balajee Kannan et al have carried out experiments using two robots to solve the problem related to autonomous recharging problem. One of the robots is assigned as working robot and the other robot assigned as recharging agent for docking and recharging. Experiment was carried out to evaluate the strategies like infinite battery, battery threshold, distance threshold, and energy-aware with mobile recharger [3].

Kuo-Lan $\mathrm{Su}$ et al have designed auto-charging system for mobile robot, with on board laser range finder assisting in detecting the landmark for docking. When the power source for the mobile robot reaches threshold level, robot 
reaches docking station for recharging with the help of laser rangefinder [4]. Carlos Antonio et al have proposed a power managing system and recharging of batteries autonomously for mobile robot. Comparison of batteries employed is also presented in this work [5].

T.Narendran et al have developed smart docking and recharging system for defense robot. In order to charge the battery of the robot, wireless charging method is implemented. Radio Frequency transmission is used for monitoring the battery status and position of the robot in the control room [6]. Roberto Quilez et al have proposed docking mobile robot in passive docks with the help of QR codes as land mark and Infrared range sensors. For docking, the robot visual pattern will assist in proper positioning with respect to the docking station [7].

Fan Guangrui et al have presented docking and recharging system based on the April Tag, which act as a reference in guiding the robot towards the vision-based docking and recharging system. The docking mechanism uses two processes for docking one is searching zone process and the other one is approaching zone process [8]. Yanan Ren et al have proposed vision feedback based docking method for intelligent wheelchair into a U-shaped bed. The docking control process consists of line trajectory tracking and path planning. An algorithm based upon identification of $U$ shaped bed which determines location and position of the wheelchair to orient into the bed by employing feedback from vision based data and dead reckoning [9].

Acosta Calderon et al have proposed solution for charging of batteries and possible method of docking station for recharging. Hardware interface in this work provides estimation of remaining capacity and state of the battery. IR sensor in the docking station and with the help of map guides the robot towards docking area. They have also tested batteries of various types [10]. Mehdi Delrobaei et al have worked on modular robots and their connection mechanism and guidance for docking and recharging. Infrared sensor assists in aligning until they are connected for recharging [11].

Roh, Se-gon et al have developed docking mechanisms using magnetic forces or friction forces with docking station and docking parts of the robot. The features of suggested technique with auto recharging have been demonstrated successfully [12]. Luo, Ren C et al have presented a system which assist in tracing sound source to track and locate destination. The robot was developed with sensors microphone, laser range finder and camera to achieve the results [13].

Jamaluddin, Anif et al have proposed system for monitoring battery for observation of current, voltage and temperature of the battery. It has been developed using microcontroller, sensors and Bluetooth module. Battery monitoring data is sent to PC using Bluetooth and Lab VIEW used for the analysis [14]. Furthermore, in [15,16], monitoring the parameters of lead acid battery using wireless local area network with the help of inter integrated circuit, universal asynchronous receiver/transmitter is proposed.

Doumbia Mamadou et al have implemented power management system monitoring the recharging process of the battery. The robot was made to dock using orientation angles and optimal positions with infrared receivers mounted on robot [17]. Priyadarshani R. Meshram et al have presented automatic docking abilities for surveillance robot. It was developed using ARM LPC2148 Controller, CMOS camera and sensors. The docking method uses ultrasonic locker and Wireless power transfer technique for recharging the robot [18].

Yi-Cheng Wu et al have presented exchanging of battery automatically and auto recharging at the docking station is presented for surveillance and security robots. A movable carrier permits \pm 9 degree of misalignment between the docking station and robot. The exchange process performed in a span of 45 seconds [19]. Ching-Chun Chuang et al in this paper presented about Battery Charger for the purpose of Industrial Mobile Robots. To get ac to dc boost converter was employed with constant-frequency control IC for to obtain stable output voltage using PWM signal [20].

Amol Deshmukh et al have in this paper have focused on management of power in autonomous robots for long duration of operation. The approach related to primary design of contemporary power management have been addressed [21].Peter Won et al have presented docking system self-reconfigurable modular mobile robots employing sensors and encoders for the estimation of orientation and distance of the modules. In this work Extended Kalman filter and particle filter state estimators were simulated to compare the results [22].

Jr-Hung Guo et al have presented multiple residual power detection system applied to mobile robot to measure the power. Microchip HOLTEK used as control core for power detection unit. It predicts the residual power using auto-regression algorithm [23]. Min Luo et al have proposed system for online battery monitoring for electric vehicles and GPRS. The goal of this system was acquiring the parameters of battery online and transferring it to PC. The system consists of main control module, data acquisition modules, GPRS module and protection module [24].

Plamen Petrov et al have presented docking station and a control method for docking of electric vehicles automatically for battery charging. Kinematics based control loops for vehicle velocity and steering angle suggested. A visual servoing system integrated with an automatic controller guiding the vehicle to dock exactly to the recharging station in a parking space $[25,26]$. Roufas et al have implemented docking mechanism employing infrared sensors and a pair of plates. Six degrees of freedom with offset sensing for estimation of angle and distance and angle between the docking plates [27]. Fan Guangrui et al have proposed docking and recharging based on vision system. The authors have used AprilTag which provide location of docking and charging device. In the docking 
method searching and approaching zones are adopted to enable proper positioning [28].

Jhzheng zhang et al have presented auto docking using ultrasonic, IR sensors and RF devices. The docking process involved wall following, coarse and accurate positioning algorithms assist in docking of the mobile robot [29]. Yanpeng Niu et al have implemented auto recharging ability for home surveillance robot. The robot was built using ARM platform Auto recharging was realized using docking using information from fusion of multiple sensors [30].

\section{PROPOSED METHODOLOGY}

In the suggested IR based docking system for autonomous recharging of mobile robot, the hardware consists of Arduino UNO Microcontroller, voltage sensor, IR sensor, Bluetooth HC 05, battery charger, ALCD Display [16x2] and 4 DC motors driven by L293 Drivers. Fig.1. Shows the block diagram of autonomous mobile robot which is programmed using an algorithm which will guide the robot to move around in a square path of 30 inches X 30 inches autonomously. Battery status in terms of peak voltage is monitored in real time by voltage detection module.

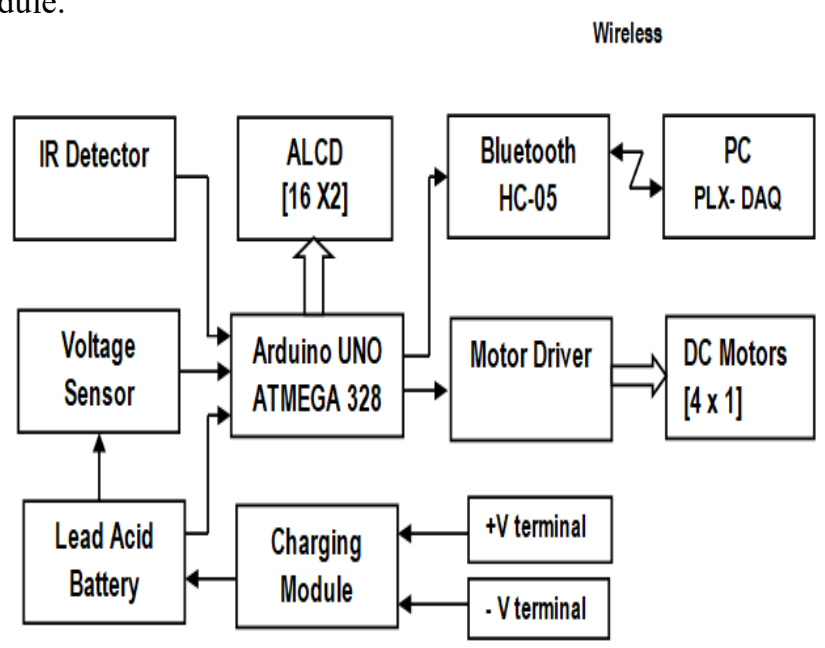

Fig. 1. Block diagram of autonomous mobile robot

The robot while carrying out its assigned task, if the battery voltage level attains threshold value, the controller directs the robot to reach the docking station for recharging autonomously. The IR receiver sensor mounted on robot gets enabled to scan for the IR transmitter sensor located besides docking station. The IR transmitter serves as a unique landmark for guiding the robot towards recharging station. If IR receiver able to detect the signal from IR transmitter the algorithm directs robot to move 10 inches straight and turn left by 90 degrees to navigate towards the docking region and connect with the docking station automatically.

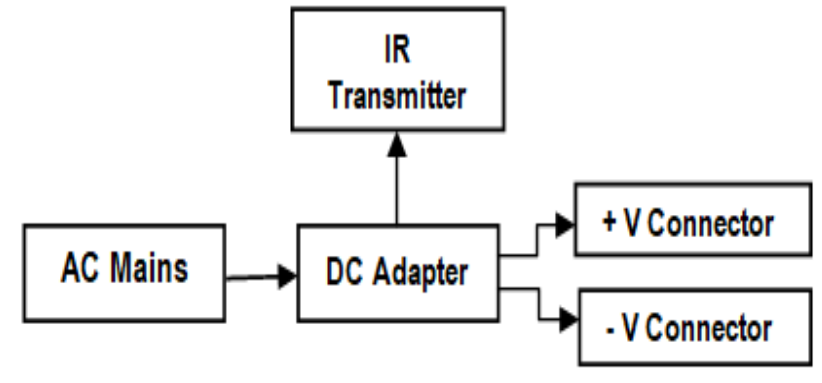

Fig. 2. Block diagram of docking station for recharging

Fig. 2 shows the block diagram of docking station which is used for recharging the robot. The robot has on board battery charger module to support charging of $12 \mathrm{~V}$ DC lead acid battery and a current sensing unit which employs a comparator to measure the differences between the input voltages to determine the charging current. The battery charging voltage values are converted to specified form, and then transferred by serial port of Arduino uno controller through HC-05 unit to the computer.

The data acquisition software tool PLX-DAQ in Microsoft excel collects voltage values from microcontroller connected to the computer and loads the data in the Excel sheet as the data approach. Also the parallax data acquisition software tool is capable of plotting the graph of acquired data in real time. Once the voltage level of the battery attains a value greater than $12 \mathrm{~V}$, the microcontroller will direct the robot to get detached from charging station and navigate back to resume the work.

\section{A. Architecture of the Proposed Autonomous Robot and the Docking Station}

The proposed four wheel-based automatic docking robot has the dimension of $25 \mathrm{~cm} \mathrm{X} 20 \mathrm{~cm} \mathrm{X} 7 \mathrm{~cm}$ ( $\mathrm{L} \mathrm{x} \mathrm{W} \mathrm{x} \mathrm{H)}$ with a rectangular structure. It consists of Arduino uno microcontroller, voltage sensor, IR sensor, Bluetooth $\mathrm{HC}$ 05, battery charger, ALCD Display [16x2] and 4 DC motors driven by L293 Drivers and sealed lead acid of 12V, $7 \mathrm{AH}$ battery. The peripheral devices are connected as shown in Fig. 3. The circuit diagrams of robot and docking station is shown in Fig. 4 and Fig. 5 respectively.

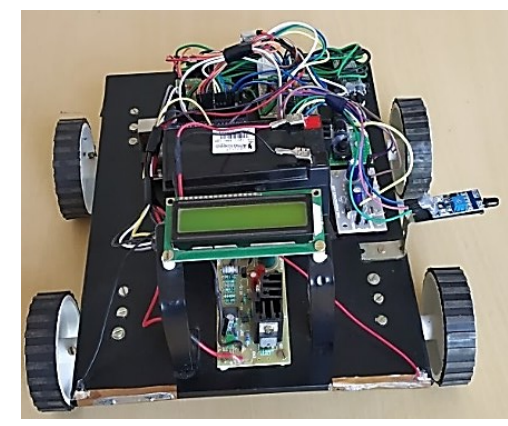

Fig. 3. Top view of automatic docking robot 


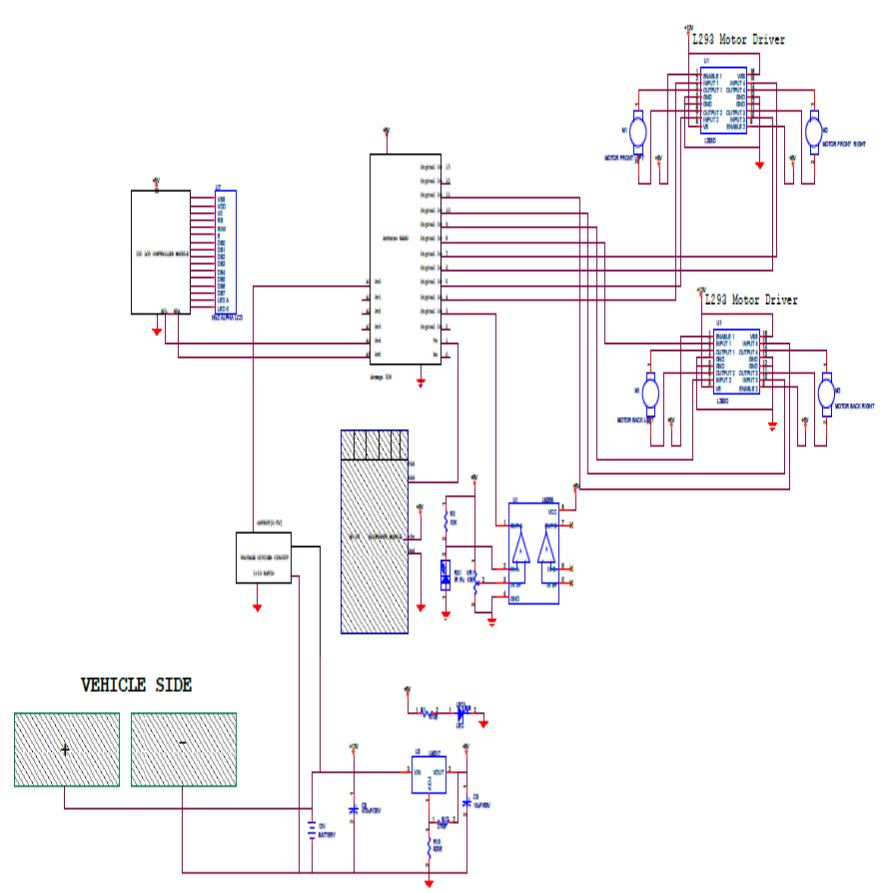

Fig. 4. Circuit diagram of the docking robot

\section{CHARGING STATION SIDE}

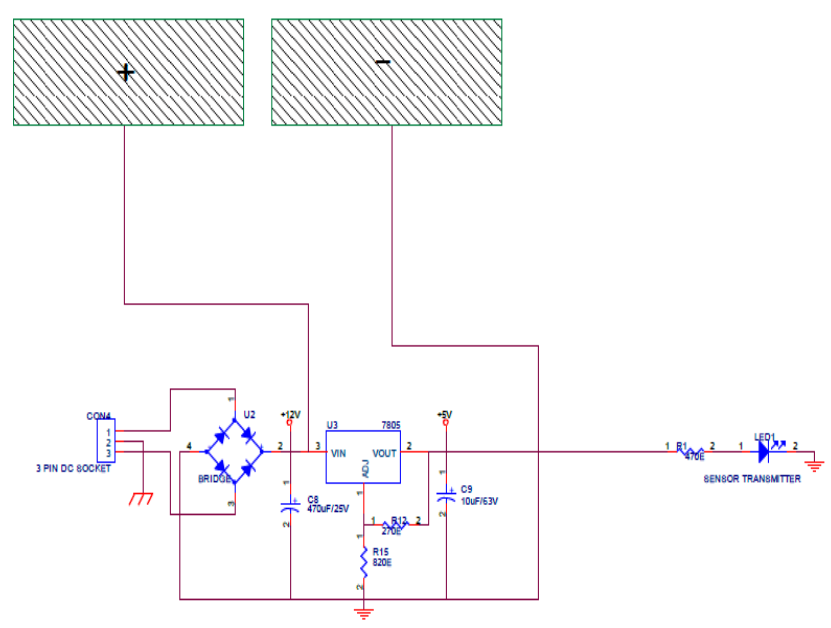

Fig. 5. Circuit diagram of the docking station

The Arduino uno microcontroller receives signals from voltage detection module about the battery voltage level and utilizes these signals to control the dc motors with the help of motor driver. The algorithm guides the robot to move around in a square path. The two H-bridge circuits control the movement and the direction of dc motors through the motor drive signals. The IR receiver sensor mounted on robot and IR transmitter sensor near docking area guide the robot in navigating towards docking area with the help of algorithm control.

The $12 \mathrm{~V}$ DC charging voltage is provided by the battery charger in the robot during recharging. At the time of battery charging, microcontroller reads the battery voltage at regular interval of time and transmits this data through $\mathrm{HC}$ -
05 Bluetooth module to the computer. PLX-DAQ software tool in the computer acquires charging data from microcontroller and stores in Excel sheet as the data arrive. The LCD display in the robot indicates the level of the battery voltage continuously.

The proposed docking and recharging station shown in Fig. 6 consists of T-shape wooden structure with flat surface mounted with metallic electrical contacts; one IR transmitter located 10 inches away from left side of the docking station as land mark which directs the robot to get docked at the recharging station. The docking station provides $12 \mathrm{~V}$ DC 1 Amp used to power the battery of the robot. In front of the robot, metallic electrical contacts are provided which establishes contact with charging station during recharging.

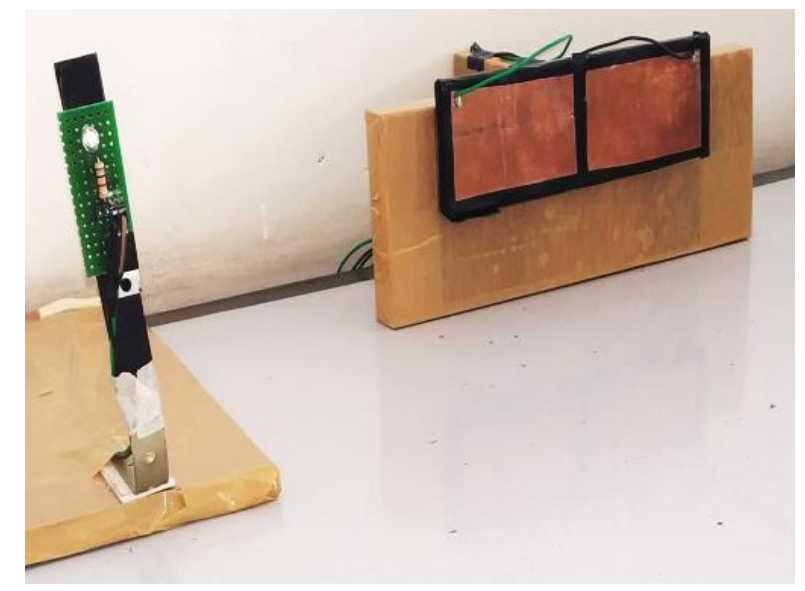

Fig. 6. IR transmitter and docking station

\section{B. Locomotion Control and Docking Method}

Wheeled robots are typically energy efficient and simple to control. A major goal of the four-wheel robot employed in the current research work is to move in a square path of 30 inch by 30 inch near the docking station controlled by the embedded program. When the IR receiver in the robot detects IR transmitter near docking area, the final docking process begins. The algorithm directs the robot to move straight by 10 inches and then turns left by $90^{\circ}$ to reach the docking area.Fig.7 shows the robot in the square path and Fig. 8 shows the robot connected to docking station.

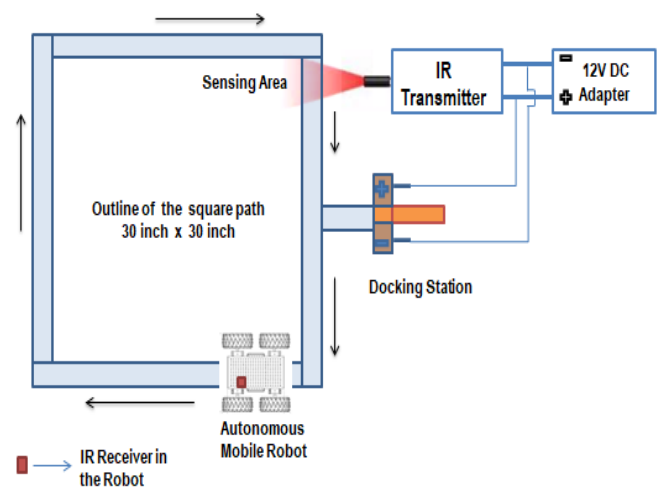

Fig. 7. Robot Moving in a Square path 


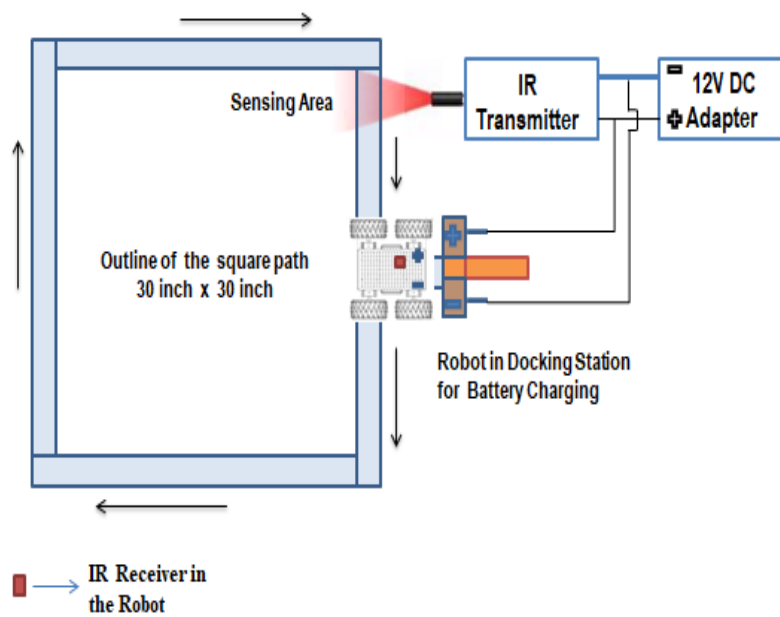

Fig. 8. Robot connected to Docking Station for Recharging programming tools.

The Arduino IDE is a cross-platform application using which the code is written in embedded $\mathrm{C}$ and uploaded to Arduino board. The parallax software tool assists in establishing communication between any device that provides serial port protocol and Microsoft Excel for acquiring the data into spread sheet. In Fig.9 the flow diagram for programming IR based auto-recharging system for autonomous mobile robot is illustrated. By initializing the Bluetooth, the charging battery voltage values are converted into required form and then transmitted through serial port of microcontroller to Bluetooth unit. The parallax software tool processes the acquired data to get tabulated in the Excel sheet.

\section{EXPERIMENTAL RESULTS}

To evaluate the proposed docking mechanism, experiment was conducted on automatic docking robot which continuously moves in its assigned path. When the battery voltage reaches $11.9 \mathrm{~V}$, the IR receiver mounted in front of the robot gets enabled. As the robot approached IR transmitter near docking station, IR receiver detects it and controller guides the robot to perform automatic docking and gets connected to the charging terminals for battery recharging.

During the process of battery charging, voltage sensor measures the voltage across battery terminals which is then converted into digital form and sent to Bluetooth communication unit through serial port of microcontroller. The serial port protocol consists of HC 05 Bluetooth unit designed for connecting serial wireless setup. The charging voltage of lead acid battery gets tabulated into columns of the Microsoft Excel sheet of the computer.

Fig.10. shows the robot connected to charging terminals in the docking station. Table. 1 presents the charging voltage values of the lead-acid battery moved to Excel sheet present in the computer. From the table it can be noted that, when robot gets connected to the charging station the voltage level in the battery was $11.89 \mathrm{~V}$ as detected by voltage sensor. Once the voltage level in the battery reaches slightly above
$12 \mathrm{~V}$, robot gets detached from docking station to move back to its original task.

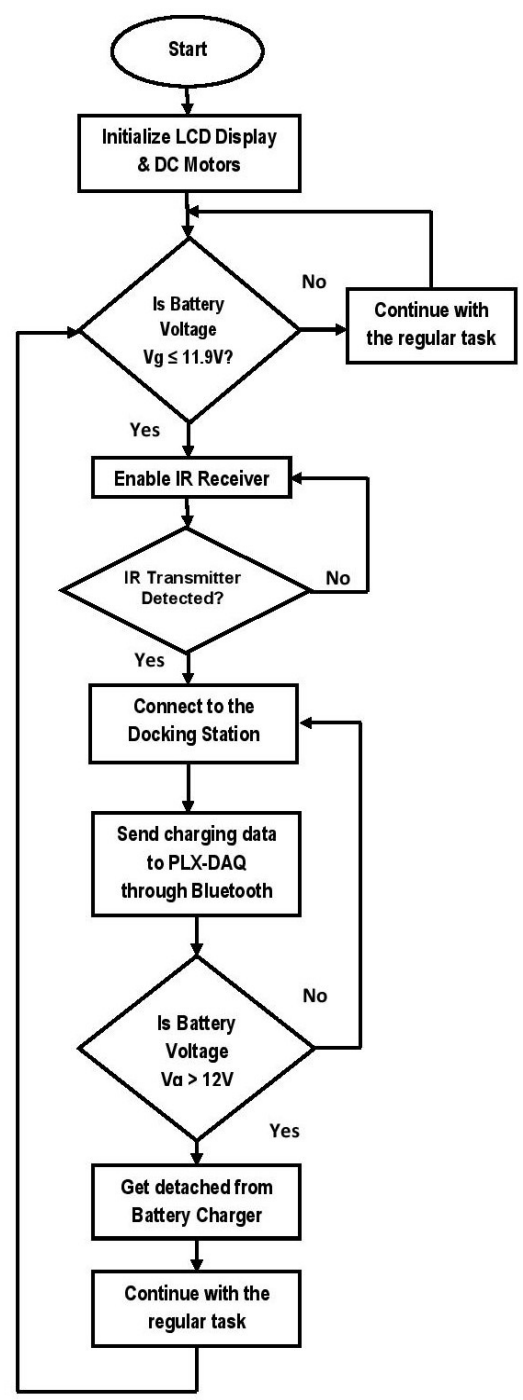

Fig. 9. Flowchart for robot docking and charging algorithm

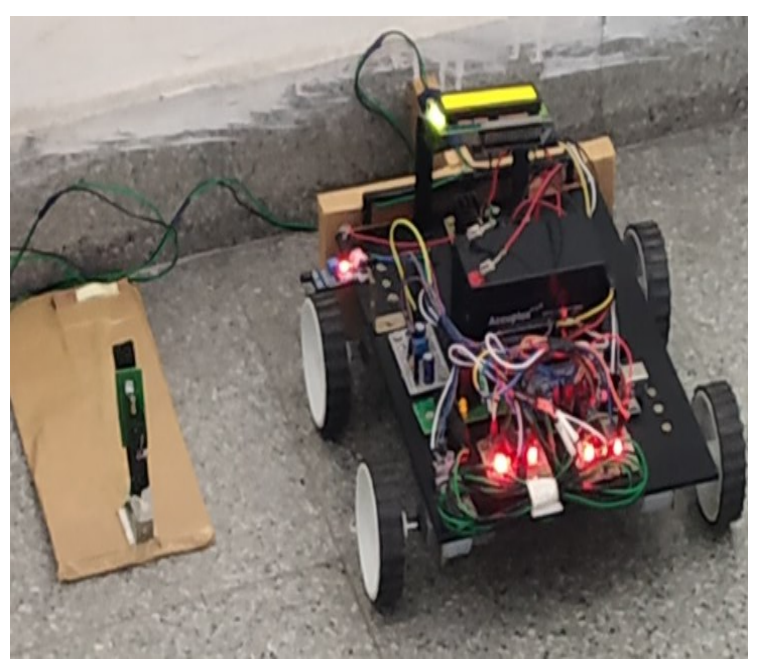

Fig. 10. Robot connected to charging terminals in the docking station 


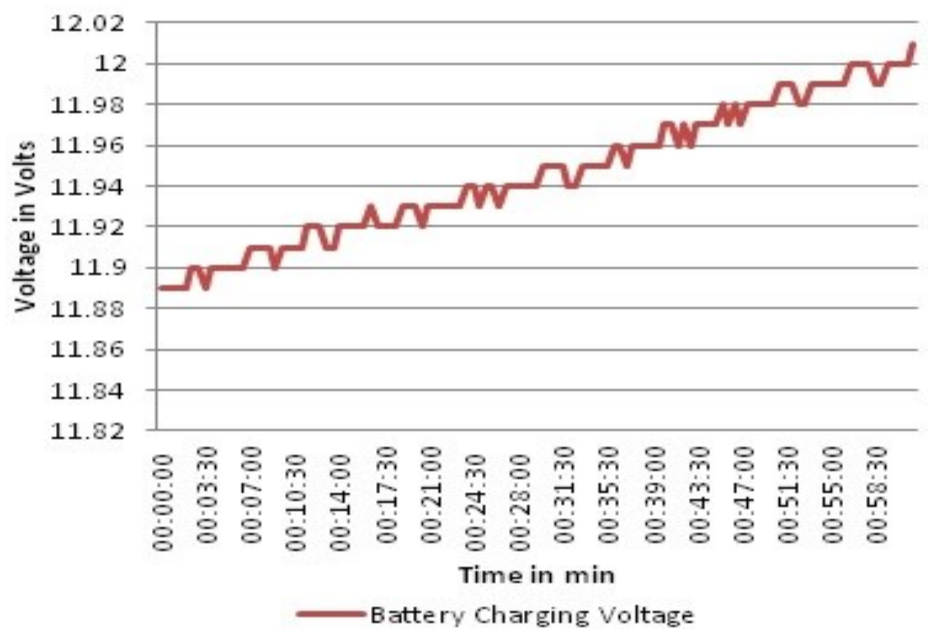

Fig. 11. Chart showing the battery charging waveform.

\begin{tabular}{|c|c|c|c|c|c|c|c|c|}
\hline $\begin{array}{l}\text { SI } \\
\text { No. }\end{array}$ & Time & $\begin{array}{c}\text { Battery Charging } \\
\text { Voltage (V) }\end{array}$ & $\begin{array}{l}\text { SI } \\
\text { No. }\end{array}$ & Time & $\begin{array}{c}\text { Battery Charging } \\
\text { Voltage (V) }\end{array}$ & $\begin{array}{l}\text { SI } \\
\text { No. }\end{array}$ & Time & $\begin{array}{c}\text { Battery Charging } \\
\text { Voltage (V) }\end{array}$ \\
\hline 1 & 00:00:00 & 11.89 & 41 & 00:20:00 & 11.93 & 81 & $00: 40: 30$ & 11.97 \\
\hline 2 & $00: 00: 30$ & 11.89 & 42 & $00: 20: 30$ & 11.92 & 82 & $00: 41: 00$ & 11.96 \\
\hline 3 & $00: 01: 00$ & 11.89 & 43 & $00: 21: 00$ & 11.93 & 83 & $00: 42: 30$ & 11.97 \\
\hline 4 & $00: 01: 30$ & 11.89 & 44 & $00: 21: 30$ & 11.93 & 84 & $00: 43: 00$ & 11.96 \\
\hline 5 & 00:02:00 & 11.89 & 45 & $00: 22: 00$ & 11.93 & 85 & $00: 43: 30$ & 11.97 \\
\hline 6 & $00: 02: 30$ & 11.9 & 46 & $00: 22: 30$ & 11.93 & 86 & $00: 44: 00$ & 11.97 \\
\hline 7 & 00:03:00 & 11.9 & 47 & 00:23:00 & 11.93 & 87 & 00:44:30 & 11.97 \\
\hline 8 & $00: 03: 30$ & 11.89 & 48 & $00: 23: 30$ & 11.93 & 88 & $00: 45: 00$ & 11.97 \\
\hline 9 & 00:04:00 & 11.9 & 49 & $00: 24: 00$ & 11.94 & 89 & $00: 45: 30$ & 11.98 \\
\hline 10 & 00:04:30 & 11.9 & 50 & $00: 24: 30$ & 11.94 & 90 & 00:46:00 & 11.97 \\
\hline 11 & 00:05:00 & 11.9 & 51 & $00: 25: 00$ & 11.93 & 91 & $00: 46: 30$ & 11.98 \\
\hline 12 & $00: 05: 30$ & 11.9 & 52 & $00: 25: 30$ & 11.94 & 92 & $00: 47: 00$ & 11.97 \\
\hline 13 & 00:06:00 & 11.9 & 53 & 00:26:00 & 11.94 & 93 & $00: 47: 30$ & 11.98 \\
\hline 14 & $00: 06: 30$ & 11.9 & 54 & $00: 26: 30$ & 11.93 & 94 & $00: 48: 00$ & 11.98 \\
\hline 15 & 00:07:00 & 11.91 & 55 & $00: 27: 00$ & 11.94 & 95 & $00: 48: 30$ & 11.98 \\
\hline 16 & 00:07:30 & 11.91 & 56 & $00: 27: 30$ & 11.94 & 96 & 00:49:00 & 11.98 \\
\hline 17 & 00:08:00 & 11.91 & 57 & $00: 28: 00$ & 11.94 & 97 & $00: 49: 30$ & 11.98 \\
\hline 18 & 00:08:30 & 11.91 & 58 & $00: 28: 30$ & 11.94 & 98 & $00: 50: 00$ & 11.99 \\
\hline 19 & 00:09:00 & 11.9 & 59 & 00:29:00 & 11.94 & 99 & $00: 51: 30$ & 11.99 \\
\hline 20 & 00:09:30 & 11.91 & 60 & $00: 29: 30$ & 11.94 & 100 & $00: 52: 00$ & 11.99 \\
\hline 21 & 00:10:00 & 11.91 & 61 & 00:30:00 & 11.95 & 101 & $00: 52: 30$ & 11.98 \\
\hline 22 & $00: 10: 30$ & 11.91 & 62 & $00: 30: 30$ & 11.95 & 102 & 00:53:00 & 11.98 \\
\hline 23 & 00:11:00 & 11.91 & 63 & $00: 31: 00$ & 11.95 & 103 & $00: 53: 30$ & 11.99 \\
\hline 24 & $00: 11: 30$ & 11.92 & 64 & $00: 31: 30$ & 11.95 & 104 & $00: 54: 00$ & 11.99 \\
\hline 25 & $00: 12: 00$ & 11.92 & 65 & $00: 32: 00$ & 11.94 & 105 & $00: 54: 30$ & 11.99 \\
\hline 26 & $00: 12: 30$ & 11.92 & 66 & 00:33:00 & 11.94 & 106 & 00:55:00 & 11.99 \\
\hline 27 & 00:13:00 & 11.91 & 67 & $00: 33: 30$ & 11.95 & 107 & $00: 55: 30$ & 11.99 \\
\hline 28 & $00: 13: 30$ & 11.91 & 68 & $00: 34: 00$ & 11.95 & 108 & $00: 56: 00$ & 11.99 \\
\hline 29 & $00: 14: 00$ & 11.92 & 69 & $00: 34: 30$ & 11.95 & 109 & $00: 56: 30$ & 12 \\
\hline 30 & $00: 14: 30$ & 11.92 & 70 & $00: 35: 00$ & 11.95 & 110 & $00: 57: 00$ & 12 \\
\hline 31 & $00: 15: 00$ & 11.92 & 71 & $00: 35: 30$ & 11.95 & 111 & $00: 57: 30$ & 12 \\
\hline 32 & $00: 15: 30$ & 11.92 & 72 & $00: 36: 00$ & 11.96 & 112 & 00:58:00 & 12 \\
\hline 33 & $00: 16: 00$ & 11.92 & 73 & $00: 36: 30$ & 11.96 & 113 & $00: 58: 30$ & 11.99 \\
\hline 34 & $00: 16: 30$ & 11.93 & 74 & 00:37:00 & 11.95 & 114 & 00:59:00 & 11.99 \\
\hline 35 & 00:17:00 & 11.92 & 75 & $00: 37: 30$ & 11.96 & 115 & $00: 59: 30$ & 12 \\
\hline 36 & $00: 17: 30$ & 11.92 & 76 & 00:38:00 & 11.96 & 116 & 01:00:00 & 12 \\
\hline 37 & 00:18:00 & 11.92 & 77 & $00: 38: 30$ & 11.96 & 117 & $01: 00: 30$ & 12 \\
\hline 38 & $00: 18: 30$ & 11.92 & 78 & 00:39:00 & 11.96 & 118 & 01:01:00 & 12 \\
\hline 39 & $00: 19: 00$ & 11.93 & 79 & $00: 39: 30$ & 11.96 & 119 & $01: 01: 30$ & 12 \\
\hline 40 & $00: 19: 30$ & 11.93 & 80 & 00:40:00 & 11.97 & 120 & 01:02:00 & 12.01 \\
\hline
\end{tabular}

The graphical programming feature in PLX DAQ software tool presents the display of charging voltage waveform of the battery as shown in Fig.11. It can be observed from the table and chart that voltage values are 
tabulated and plotted at successive intervals of $30 \mathrm{sec}$. When the battery is charged greater than $12 \mathrm{~V}$ microcontroller stop charging, this is represented in the graphical display. The advantage of recording battery charging voltage and its plot helps in analyzing the battery charging process and estimated time required to charge the battery to $12 \mathrm{~V}$ using $1 \mathrm{Amp}$ charger. The charging time can be reduced by connecting to higher current rating chargers which helps the robot to resume its original task quickly.

\section{CONCLUSION}

The proposed automatic docking robot is designed and developed successfully for autonomous docking and recharging. A docking technique on the basis of algorithm which guides the robot to move around in a square path of 30 inch by 30 inch continuously and the IR detector near the docking station is suggested. When the on-board battery is low, the robot proceeds back to the docking station for recharging operations. The robot accomplished a success rate of $95 \%$ for the various docking attempts. Future work can focus on improving the robot employed in the current work to accommodate more features. It can be planned to address few specialized technical issues such as adding more docking stations, the automatic battery replacement mechanism and visual navigation.

\section{REFERENCES}

[1] Raj, K. Varun, et al. "A beacon-based docking system for an autonomous mobile robot." Proceedings of the 13th National Conference on Mechanisms and Machines (NaCoMM07). 2007

[2] Song, Guangming, et al. "Automatic docking system for recharging home surveillance robots." IEEE Transactions on Consumer Electronics 57.2 (2011): 428-435.

[3] BalajeeKannan, Victor Marmol, Jaime Bourne and M. Bernardine Dias, The Autonomous Recharging Problem: Formulation and Market-based Solution, IEEE International Conference on Robotics and Automation (ICRA)Karlsruhe, Germany, May 6-10, 2013

[4] Kuo-Lan Su1, Yi-Lin Liao2, Shih-Ping Lin3 and Sian-Fu Lin3, "An Interactive Auto-recharging System for Mobile Robots"(Received 2 March 2013; Accepted 3 May 2013; Published on line 1 March 2014)

[5] Carlos Antonio Acosta Calderon, Buck Sin NG,Elara Rajesh Mohan and HengKhai NG, Docking System and Power Management for Autonomous Mobile Robots, Applied Mechanics and Materials Vol. 590 June, 2014

[6] Narendran.1,Mr.P.Saravanan.2,Dr.V.Nandagopa, Design and Implementation of Smart Docking and Recharging System for Defense Robot, IOSR Journal of Electrical and Electronics Engineering (IOSR-JEEE) e- ISSN: 2278-1676,p-ISSN: 23203331, Volume 10, Issue 1 Ver. II (Jan - Feb. 2015).

[7] Roberto Quilez, Adriaan Zeeman, Nathalie Mitton, \& Julian Vandal, Docking autonomous robots in passive docks with Infrared sensors and QR codes, International Conference on Test beds and Research Infrastructures for the Development of Networks \& Communities (TridentCOM), Jun 2015, Vancouver, Canada. 2015

[8] Fan Guangrui, Wang Geng, Vision-Based Autonomous Docking and Re-charging System for Mobile Robot in Warehouse Environment, 2nd International Conference on Robotics and Automation Engineering, 2017

[9] YananRen, Wei Zou, Hao Fan, Aixue Ye and Kui Yuan, “ A docking control method in narrow space for intelligent wheelchair", Proceedings of 2012 IEEE International Conference on Mechatronics and Automation, August 5 - 8, Chengdu, China

[10] Acosta Calderon, Antonio Carlos, Buck Sin Ng, Elara Rajesh Mohan, and HengKhai Ng. "Docking system and power management for autonomous mobile robots." In Applied
Mechanics and Materials, vol. 590, pp. 407-412. Trans Tech Publications Ltd, 2014.

[11] Mehdi Delrobaei, Qeshm Tehran, Kenneth A. McIsaac "An Infrared Docking System for Modular Wheeled Mobile Robots,"In Applied Mechanics and Materials, vol. 590, pp. 407412. Trans Tech Publications Ltd, 2014.

[12] Roh, Se-gon, Jae Hoon Park, Young Hoon Lee, Young Kouk Song, KwangWoong Yang, Moosung Choi, Hong-Seok Kim, Hogil Lee, and HyoukRyeol Choi. "Flexible docking mechanism with errorcompensation capability for auto recharging system of mobile robot." International Journal of Control, Automation, and Systems 6, no. 5 (2008): 731-739.

[13] Luo, Ren C., Chien H. Huang, and Chun Y. Huang. "Search and track power charge docking station based on sound source for autonomous mobile robot applications." In 2010 IEEE/RSJ International Conference on Intelligent Robots and Systems, pp. 1347-1352. IEEE, 2010.

[14] Jamaluddin, Anif, Fengky Adie Perdana, AgusSupriyanto, AgusPurwanto, and M. Nizam. "Development of Wireless Battery Monitoring for electric vehicle." In 2014 International Conference on Electrical Engineering and Computer Science (ICEECS), pp. 147-151. IEEE, 2014

[15] Saputra, Oka Danil, SeogChae, Young Hyung Kim, and Soo Young Shin. "Remote Monitoring of Lead-ACID BATTERY BASED ON WLAN." Proceedings of the 3rd International Conference on Industrial Application Engineering 2015.

[16] Rauniyar, Ashish, Mohammad Irfan, Oka DanilSaputra, Jin Woo Kim, Ah Ra Lee, Jae Min Jang, and Soo Young Shin. "Design and Development of a Real-Time Monitoring System for Multiple Lead-Acid Batteries Based on Internet of Things." Future Internet 9 , no. 3,2017.

[17] Doumbia, Mamadou, Xu Cheng, and Vincent Havyarimana. "An auto-recharging system design and implementation based on infrared signal for autonomous robots." In 2019 5th International Conference on Control, Automation and Robotics (ICCAR), pp. 894-900. IEEE, 2019.

[18] Priyadarshani R. Meshram, S. P. Tondare " Automatic Docking System for Home Surveillance Robots," International Journal of Scientific \& Engineering Research, Volume 7, Issue 5, May-2016

[19] Wu, Yi-Cheng, Ming-Chang Teng, and Yi-Jeng Tsai. "Robot docking station for automatic battery exchanging and charging." In 2008 IEEE International Conference on Robotics and Biomimetics, pp. 1043-1046. IEEE, 2009.

[20] Chuang, Ching-Chun, Chih-Wei Chuang, Chih-Chiang Hua, and Sen-Tung Wu. "Development of Battery Charger for Industrial Mobile Robots." In 2019 IEEE Eurasia Conference on IOT, Communication and Engineering (ECICE), pp. 449-452. IEEE, 2019

[21] Deshmukh, Amol, Patricia A. Vargas, Ruth Aylett, and Keith Brown. "Towards socially constrained power management for long-term operation of mobile robots." TAROS 2010

[22] Won, Peter, Mohammad Biglarbegian, and William Melek. "Development of an effective docking system for modular mobile self-reconfigurable robots using extended kalman filter and particle filter." Robotics 4, pp. 25-49. 2015.

[23] Guo, Jr-Hung, Jie-Tong Zou, and Kuo-Lan Su. "Development of the residual power prediction system for mobile robots." Artificial Life and Robotics 17, no. 1, 24-29, 2012.

[24] Luo, Min, Yong Xiao, Wei-Ming Sun, and Zhiping Wang. "Online battery monitoring system based on gprs for electric vehicles." In 2013 5th International Conference on Intelligent HumanMachine Systems and Cybernetics, vol. 1, pp. 122-125. IEEE, 2013.

[25] Petrov, Plamen, Clément Boussard, SamerAmmoun, and FawziNashashibi. "A hybrid control for automatic docking of electric vehicles for recharging." In 2012 IEEE International Conference on Robotics and Automation, pp. 2966-2971. IEEE, 2012.

[26] Pérez, Joshué, FawziNashashibi, Benjamin Lefaudeux, Paulo Resende, and Evangeline Pollard. "Autonomous docking based on infrared system for electric vehicle charging in urban areas." Sensors 13, no. 2, 2645-2663, 2015

[27] Roufas, Kimon, Ying Zhang, Dave Duff, and Mark Yim. "Six degree of freedom sensing for docking using IR LED emitters and 
receivers." In Experimental Robotics VII, pp. 91-100. Springer, Berlin, Heidelberg, 2001.

[28] Guangrui, Fan, and Wang Geng. "Vision-based autonomous docking and re-charging system for mobile robot in warehouse environment." In 2017 2nd International Conference on Robotics and Automation Engineering (ICRAE), pp. 79-83. IEEE, 2017.

[29] Juzhong Zhang, Liming Cai, YuyiChu,Qixun, Zhon “ A Sectional Auto-docking Charging Control Method for the Moble Robot,"
Proceedings of 2019 IEEE International Conference on Mechatronics and Automation August 4 - 7, Tianjin, China,2019.

[30] Niu, Y. , Zhang, J. , Meng, T. , \& Wang, H. "Design of a home surveillance robot with self-recharging capabilities," International Symposium on Knowledge Acquisition \& Modeling. IEEE., 2010. 